EGU2020-17874

https://doi.org/10.5194/egusphere-egu2020-17874

EGU General Assembly 2020

(c) Author(s) 2020. This work is distributed under

the Creative Commons Attribution 4.0 License.

\title{
What controls nucleation of ice and nitric acid hydrates by meteoric material?
}

\author{
Alexander James ${ }^{1}$, Sebastien N. F. Sikora ${ }^{2}$, Mark Holden³, Graham W. Mann², John M. C. Plane', \\ and Benjamin J. Murray ${ }^{2}$ \\ ${ }^{1}$ University of Leeds, School of Chemistry, Leeds, United Kingdom of Great Britain and Northern Ireland \\ (a.james1@leeds.ac.uk) \\ ${ }^{2}$ University of Leeds, School of Earth and Environment, Leeds, United Kingdom of Great Britain and Northern Ireland \\ ${ }^{3}$ University of Central Lancashire, School of Physical Sciences and Computing, Preston, United Kingdom of Great Britain and \\ Northern Ireland
}

\begin{abstract}
Nucleation of crystalline ice and nitric acid hydrates in Polar Stratospheric Clouds (PSC) is important for the destruction of ozone, both through changing the rate of activation of ozone destroying species and through the removal by sedimentation of nitric acid, which can deactivate ozone destroying species. Nucleation is thought to proceed heterogeneously on fragmented meteoric materials, leading to formation of ice and nitric acid trihydrate. The heterogeneous nature of meteoric materials and the potential to form multiple crystalline phases makes this system particularly complex. In particular, the characteristics of meteoric fragments which allow them to nucleate crystallisation in PSCs are unknown. We have investigated the nature of nucleation of nitric acid solutions on meteorite thin section surfaces. We find that nucleation occurs on a range of sites on the surface without significant reproduction in repeat freezing experiments. Electron microscopy showed significant diversity in the type of surface features present in regions where nucleation was observed. This is in contrast to recent studies of ice nucleation on K-feldspar and quartz surfaces, where particular sites were found to dominate nucleation. We also observed a range of different crystalline phases forming competitively, some of which are not represented on the $\mathrm{HNO}_{3} / \mathrm{H}_{2} \mathrm{O}$ equilibrium phase diagram. The results reinforce the complexity of nucleation in PSC and do not support simplifying assumptions commonly made in the literature e.g. around the order in which phases form. In order to facilitate a predictive capacity of future trends in ozone loss significant work is required in understanding the nucleation of nitric acid hydrates by meteoric material.
\end{abstract}

